\title{
Chronic Sinusitis with Frontal Mucocele: A Case Report
}

\author{
D. S. Deenadayal*, B. Naveen Kumar, K. Lakshmi Sameeri \\ Department of Otorhinolaryngology, Yashoda Hospital, Secunderabad, India \\ Email: ${ }^{\text {aarticlinic@yahoo.com }}$
}

Received 26 February 2014; revised 25 March 2014; accepted 24 April 2014

Copyright (C) 2014 by authors and Scientific Research Publishing Inc.

This work is licensed under the Creative Commons Attribution International License (CC BY).

http://creativecommons.org/licenses/by/4.0/

\section{(c) (i) Open Access}

\begin{abstract}
Purpose: To describe a type IIIa frontal mucocele in a case of diffuse nasal polyposis. To discuss the clinical presentation, diagnostic and treatment modalities. Study Design: A case report including radiological, histopathological analysis and review of literature. Methods: A case report from a tertiary care centre. Histopathologic and radiological details are reviewed. Results: This case report presents a 59-year-old male who came to us with fronal headaches and nose blocks of 6 months' duration. Clinical examination revealed bilateral nasal polyposis. Plain CT scan of paranasal sinuses revealed a circumscribed lesion with erosion of inner table of left frontal sinus amidst pan sinus haziness. We addressed the condition by combined FESS with eyebrow incision frontal craniotomy. Histopathological examination revealed a mucocele. Conclusion: In combined approach, eyebrow incision minimal frontal craniotomy remains the optimal option for visualisation of entire sinus, especially in laterally placed frontal mucoceles; while FESS helps in maintaining physiological drainage and proper follow-up.
\end{abstract}

\section{Keywords}

Chronic Sinusitis

\section{Introduction}

Mucoceles are mucus containing, epithelial lined, locally expansile lesions, capable of causing erosion and remodelling of the containing sinus wall [1]. Frontal mucoceles are the most common, contributing $60 \%-80 \%$ of all. Posterior table of the sinus is thinner compared to the anterior wall resulting in early destruction and intracranial extension.

${ }^{*}$ Corresponding author. 


\section{Case Report}

A 59-year-old male patient approached us with nose blocks, frequent colds and left sided frontal headaches of 6 months' duration. Clinical examination was showing deviated septum and polyposis of both nasal cavities. Patient was evaluated with CT PNS with and without contrast, which showed pan sinus haziness with loss of haustrations and full thickness erosion of part of inner wall of left frontal sinus (Figure 1). A fracture line of forgotten trauma was also identified.

Patient was posted for surgery under general anaesthesia. Bilateral debrider assisted FESS was done. Polypoid tissue was cleared from frontal recess. In view of visualising lateral part of sinus, we opted for Left frontal craniotomy with eyebrow incision. A $4 \mathrm{~cm}$ bony window was created in the anterior table. Polypoid tissue was cleared from left frontal sinus. Continuity with frontal recess achieved. $3 \mathrm{~cm}$ defect found in posterior table (Figure 2). Dural intactness confirmed and glue applied. Sinus was packed with Triamcinalone soaked gelfoam. Anterior sinus wall reconstructed with miniplates.

Histopathological examination of the tissue obtained from left frontal sinus revealed chronic sinusitis with features suggestive of mucocele (Figure 3).

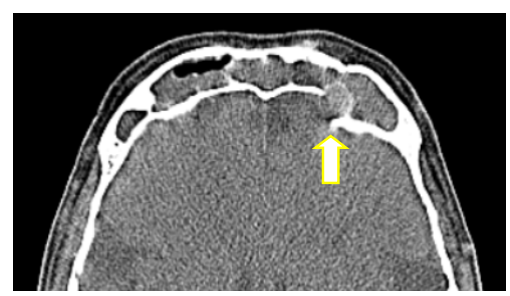

Figure 1. Type 3a mucocele left frontal sinus, showing erosion of posterior table.

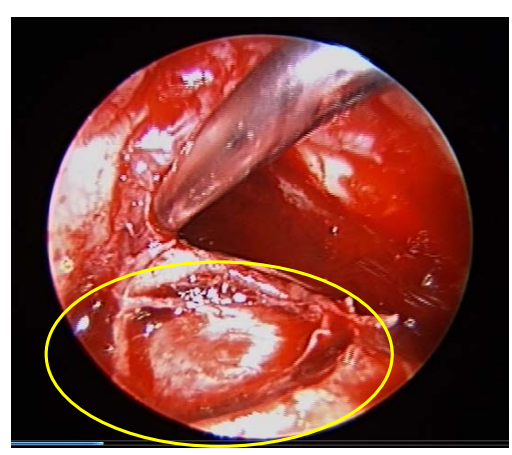

Figure 2. Erosion of posterior table, exposing dura (7 o'clock position).

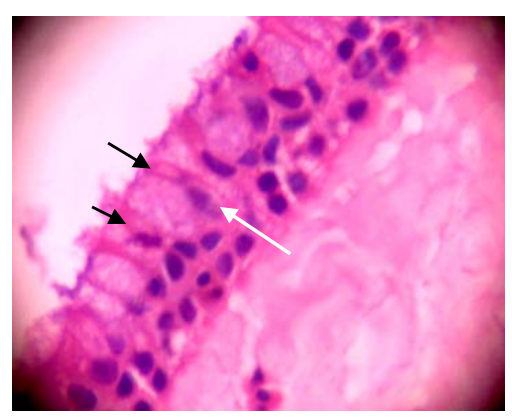

Figure 3. Epithelial lining of mucocele showing pseudostratified columnar epithelium (white arrows) and hypertrophic goblet cells (black arrow). 
After four weeks of follow-up, patient had a trauma over the surgery site, started complaining of discharge from the wound area, where we observed extrusion of bony sequestrum. Re-exploration was done under local anaesthesia, and the disease in the sinus was found to be resolved with proper drainage of the sinus.

Wound healing was good after removal of all sequestra, with negligible change in the contour of the supraorbital rim.

\section{Discussion}

Mucoceles can be primary, when they are developed due to blockage of the natural ostium, where they fill the entire sinus, or secondary, when they are developed due to entrapment of mucosa, as in fractures, which can be localised.

They have bone eroding and remodelling properties, due to the osteolytic enzymes-IL-1, 6, TNF alpha, PGE-2 secreted by lining epithelium, stimulation of osteoclasts and the exerted intra mucocele pressure on the surrounding wall.

Frontal mucoceles have been classified into 5 types depending on the extent [2].

Type I: Mucocele limited to the frontal sinus only with or without orbital extension.

Type II: Mucocele is found involving the frontal and ethmoidal sinuses with or without orbital extension.

Type IIIa: Mucocele erodes the posterior wall of the frontal sinus with minimal or no intracranial involvement.

Type IIIb: Mucocele erodes the posterior wall with major intra cranial extension.

Type IV: Mucocele erodes the anterior wall of the frontal sinus.

Type Va: There is erosion of both anterior and posterior walls of frontal sinus without or minimal intracranial extension.

Type Vb: There is erosion of both anterior and posterior walls of frontal sinus with a major intracranial extension.

\section{Conclusions}

Our patient had 4 issues-

1) Forgotten trauma of frontal bone.

2) Severe nasal polyposis.

3) Relatively small mucocele eroding posterior table.

4) Trauma causing necrosis of the plate fixed frontal bone with sequestrum formation.

Though chronic sinusitis and nasal plyposis contribute to formation of mucoceles in majority of cases, craniofacial trauma is reported to cause approximately $28 \%$ of mucoceles. Frontal sinus is the most common site owing to its complex drainage.

Symptoms may range widely in severity, even intracranial extension causing minimal symptoms. There were case reports describing meningitis developed in a mucocele with intracranial extension involving dura.

Radiological evaluation helps not only in precise understanding of the anatomy, extension of disease, but also in diagnosing involvement of dura and planning of surgery.

Though functional endoscopic sinus surgery evolved a lot in managing severe sinus disease, combined approach is still optimal choice aiding in complete removal of pathological tissue, restoration of drainage pathway and also cosmetically acceptable scar. It is reliable in cases of polyposis and has advantages of checking intactness of dura, reduced CSF leak and repair if necessary and also reduced need for revision surgeries.

Cranialisation or obliteration of sinus was not considered in our case, as we achieved a good drainage pathway that enables accurate follow-up. Along with collaboration with a neurosurgeon, navigation technology aids the anatomical knowledge and surgical technique and has the potential to improve efficiency \& safety of the surgery.

\section{References}

[1] Gavioli, C., Grasso, D.L., Carinci, F., Amoroso, C. and Pastore, A. (2002) Review Mucoceles of the Frontal Sinus. Clinical and Therapeutical Considerations. Minerva Stomatologica, 51, 385-390.

[2] Har-El, G. (2001) Transnasal Endoscopic Management of Frontal Mucoceles. Otolaryngologic Clinics of North America, 34, 243-251. http://dx.doi.org/10.1016/S0030-6665(05)70309-1 